\title{
LOS EFECTOS ADVERSOS DE LA MINERÍA EN LA ECONOMÍA
}

\author{
THE ADVERSE EFFECTS OF MINING IN THE ECONOMY \\ Nicko Alberto Gomero Gonzales* \\ Docente Principal de la Facultad de Ciencias Contables \\ Víctor Ricardo Masuda Toyofuku** \\ Docente Auxiliar de la Facultad de Ciencias Contables \\ Universidad Nacional Mayor de San Marcos - UNMSM / Lima - Perú \\ [Recepción: Setiembre de 2015/ Conformidad: Octubre 2015]
}

\section{RESUMEN}

Los países que han sustentado su desarrollo basado en la innovación, el conocimiento, ciencia y tecnología, han logrado resultados mucho más favorables que aquellos que solo han centrado sus resultados macro y microeconómicos en la actividad extractiva como es la minera. La volatilidad de este sector y su anclaje al escenario internacional condiciona que los indicadores de crecimiento económico también tengan el mismo comportamiento. Es decir, su sostenibilidad está correlacionada a las señales de la economía global, especialmente de aquellos países demandantes de materias primas. Es más, los gastos e inversiones en proyectos sociales como los mismos recursos presupuestales, están supeditados a los vaivenes de los precios internacionales de los minerales. Asimismo, existen estudios que han evidenciado que los gobiernos con mayor grado de corrupción, son aquellos en donde se observa la abundancia de recursos naturales que dicho sea de paso, también se constituyen en el punto de partida para las convulsiones sociales.

\section{Palabras clave:}

Minería; economía; volatilidad; proyectos sociales.

\begin{abstract}
The countries that have supported its development based on innovation, knowledge, science and technology have achieved much more favorable results than those only having focused their macro and micro economy in extraction activities such as mining. The instability of this sector and its attachment to the international scene determines that the indicators of economic growth also have the same behavior. That is, its sustainability is correlated to signs of the global economy, especially those countries demanding raw materials. Moreover, expenses and investments in social projects such as the same budgetary resources, are subject to the vagaries of international prices of minerals. Furthermore, studies have shown that governments with more corruption, are those with abundant natural resources, which by the way, also constitute the starting point of the social convulsions observed.
\end{abstract}

Keywords:

Mining; economy; volatility; social projects.

\footnotetext{
* Doctor en Ciencias Económicas. Magíster en Contabilidad con mención en Banca y Finanzas -UNMSM. Email: gomero_econ@yahoo.es

** Máster en Tributación y Política Fiscal. Email: vmasudato@gmail.com
} 


\section{INTRODUCCIÓN}

La redistribución de la riqueza está en función de cuanto sea el crecimiento económico. Las políticas sociales que ensayan los gobiernos no pueden intentar disminuir las brechas sociales, si es que antes no logran que los indicadores macro económicos alcancen buenos resultados; en especial, el PBI y con ello, el ingreso per cápita. Para que la economía avance, es necesario que las políticas económicas que se implementen sean coherentes, prudentes, serias, entre otras condiciones y que, no estén contaminadas de intencionalidades "perversas" que solo buscan beneficios personales poniendo en un segundo plano, los logros que necesita la sociedad.

La política económica, como son la fiscal, la monetaria y la cambiaria, combinadas con las políticas públicas, deben de crear un escenario adecuado para que los actores económicos converjan y sumen esfuerzos para potenciar el crecimiento económico.

La creación de un entorno, en donde están presentes los factores económicos, sociales, políticos debe de minimizar todo tipo de riesgo e incertidumbre de tal forma que, los encargados de crear riqueza como son las empresas, actúen bajo las mejores condiciones para sobreponerse así a los retos que imponen los mercados. Asimismo, la minimización de las vulnerabilidades tiene que apuntar a generar espacios en donde las inversiones generen los mayores efectos multiplicadores económicos y sociales.

Todo gobierno maneja una agenda económica y social, en donde están presente la política económica en todas sus dimensiones; es decir, aquellas que están direccionadas a terminar con los indicadores de pobreza o en todo caso, todas aquellas "taras" que se derivan de la ineficiente asignación de los recursos. Como argumentan los teóricos, las asimetrías sociales no son un resultado "perse" sino que, se fundamenta en las posiciones equivocadas de aquello que tienen el poder de influir en el manejo de los recursos económicos.

Las evidencias señalan que la corrupción está mayormente focalizada en aquellas sociedades en donde se aprecian profundas asimetrías sociales y económicas, siendo uno de los indicadores de medición, la pobreza. El presente estudio se plantea como objetivo demostrar cómo la decisión de querer crecer en base de la extracción de recursos naturales, en el largo plazo solo generará crecimientos económicos lentos y pocos sostenidos complementados conn convulsiones sociales.

No existe un país con riqueza suficiente para redistribuir, si es que antes no se ha logrado, fortalecer las estructuras económicas. El logro de este objetivo está anclado a un factor que en estos últimos años, se ha convertido en una pieza clave de desarrollo sostenido a largo plazo, el cual es la educación. Las productividades marginales que se observan en cada país están correlacionadas directamente con los factores tecnológicos y de la calidad educativa, afirmación que se evidencia con las estadísticas que se observan en los países más desarrollados, en donde su grado de competitividad global está correlacionado a estos pilares básicos.

País que decide potenciar el pilar de la educación, conjuntamente con la innovación y tecnología, fundamentará su crecimiento en la industria de la inteligencia competitiva, en el sector de alta tecnología, en la actividad satelital; es decir, en aquellos sectores donde se requiere conocimientos que se derivan de la actividad científica. Estos países son los que en un futuro tendrán la supremacía y hegemonía mundial.

Por otro lado, están los que basan su supuesto desarrollo en la actividad extractiva en donde el producto final no tiene valor agregado alguno, y en donde la actividad industrial es solo una franja marginal en la actividad productiva y cuyo aporte al crecimiento económico es débil y poco sostenido. Escenario que se complica cuando los actores económicos se convierten en participantes directos de las convulsiones sociales, que al final termina por contaminar a toda la economía. País que centra su desarrollo en esta actividad productiva, podrá crecer, pero no tanto como lo haría apostando por actividades alternativas, cuya materia prima principal es la ciencia y tecnología que por cierto van amarrada a la calidad educativa.

Basado en esta última teoría se encuentran los países que anclan su economía a la actividad minera, que en realidad se constituye en la locomotora de la mayor parte de su actividad económica y financiera. Los ingresos tributarios, los gastos sociales, las plani-

88/ QVIPURAMAYOC | Vol. 23(44) 2015 
llas del Estado y la puesta en marcha de las políticas redistributivas, se encuentran enmarcados a los ingresos que puedan provenir de las exportaciones de minerales.

La mezcla de exportación, $80 \%$ de productos mineros y el $20 \%$ con valor agregado, más que una fortaleza se constituye en un riesgo para estas economías, ya que su viabilidad económica está altamente correlacionada con el comportamiento macroeconómico de los países industrializados. Si estas últimas crecen sostenidamente, vía precios, las economías especializadas en extraer y exportar concentrados vivirán en abundancia, pero si estas se volatilizan con tendencia a la recesión, sentirán los síntomas de la pobreza. Este es el perfil clásico de estas economías, que como se manifestó, todo su proceso se sostiene en un sector de alta volatilidad, como es la minería.

Cabe precisar que Joseph Stiglitz, el 2007, conjuntamente con Jeffrey Sachs y Macartan Humprhreys, publicaron el libro "Escapando de la maldición de los recursos naturales" en donde llegaron a demostrar que los países que son ricos en recursos naturales como los poseedores de petróleo, han tenido un proceso de crecimiento más lento que aquellos países que han basado su desarrollo en tecnología, conocimiento, investigación; es decir, en todos aquellos factores que sustentan la industria tecnológica o de alto contenido agregado.

Bajo esta misma corriente de investigación, Jeffre Sachs y Andrew Warner (1997), tomando en cuenta un conjunto de países, llegaron a determinar una correlación inversa entre la cantidad de recursos naturales y el crecimiento económico. El argumento de estos economistas se centra en que la dependencia de un país a ciertos productos cuyos precios depende de las volatilidades del mercado mundial es débil y poco sostenida.

Como se comentó, en espacios económicos en donde los recursos naturales están concentrados y listos para su explotación, van a converger una serie de intereses de grupo y particulares que van actuar como palanca para la aparición de las convulsiones sociales, hechos focalizados por un tiempo pero que luego, se van a multiplicar.
Tal como lo señala Ferrufino Rubén (2007). La teorización sobre la maldición de los recursos naturales se encuentran en cuatro pilares básicos: a) la enfermedad holandesa, b) la persecución de la renta, c) la volatilidad en el flujo de la renta de los recursos naturales asociada a la inestabilidad de los precios internacionales y d) la paradoja de la abundancia.

En los pilares básicos mencionados por el autor, cabe destacar el segundo punto, llamado persecución de la renta, donde explica y señala, que en economías en donde existe la concentración de recursos, la población hace menos esfuerzos para generar riqueza, denominador común en muchos países, incluyendo la peruana, que en estos últimos años sucumbió a los efectos de esta paradoja económica.

A la luz de esta teoría, se precisa que los gobiernos, como gozan de las rentas provenientes de la actividad extractiva de los recursos naturales, solo inyectan fondos al circuito económico y financiero de los que provienen de esta actividad, dejando de lado el esfuerzo de generar por otras formas de rentas permanentes.

\section{RESULTADOS DE LA INVESTIGACIÓN}

\section{Análisis de cifras globales}

Las cifras que reflejan el proceso de crecimiento económico a nivel mundial presentan importante asimetría entre los países cuyos gobiernos inclinaron sus políticas públicas y económicas al desarrollo industrial o en todo caso, a la del conocimiento y, por otro lado, aquellos que basaron su modelo de desarrollo en el andamiaje de la industria sin valor agregado, llamados extractivos, como son los minerales. Los resultados son totalmente diferenciados, los países industrializados, cuyos pilares básicos de desarrollo se basan en la educación, investigación, tecnología e innovación permanente, entre otros factores propios de una industria competitiva, lograron cifras de PBI per cápita que oscilan en un rango de los $\$ 40000$ y $\$ 70000$, entre los cuales se encuentran Noruega, Estados Unidos, Finlandia, Japón. En el otro lado, de la orilla están Bolivia, Perú, Chile, Colombia, países que creen todavía que estar anclados a los commodities es la solución a los problemas económicos y sociales, 
hecho que fundamenta el por qué sus gobiernos, en sus agendas de gestión social y económica, los pilares básicos que potencian el crecimiento sostenido como es la calidad educativa, es tomado como un elemento marginal.

Tal como argumentan los teóricos, los países supeditados a sus materias primas, las volatilidades de sus indicadores macroeconómicos están correlacionadas a lo que suceda en los escenarios internacionales. Países como Bolivia, Perú, Venezuela, Ecuador, que cuentan con ingentes recursos naturales como mineros, petroleros y ahora con los yacimientos de gas, más que fortalezas, presentan debilidades en sus estructuras económicas. Si se aprecia sus exportaciones, como promedio los industrializados solo representa entre el $20 \%$ al $30 \%$ y con mercados poco diversificados, a esto hay que complementar las convulsiones sociales que se presentan en los espacios geográficos en donde están localizados estos recursos, bajo el pretexto de la contaminación ambiental o responsabilidad social.

Estos conflictos que suceden en serie, no solo afecta al sector extractivo; sino a todo el país, debido a que los inversionistas globales no segmentan los riesgos solo que toman como referencia el Emerging Market Bond Index (EMBI) de cada país que es evaluado para decidir una inversión.

En el gráfico $\mathrm{N}^{\circ} 01$ refleja lo afirmado, en donde se aprecia la total asimetría en la distribución de la riqueza global y que se concentra en aquellos países que han logrado su desarrollo a base de un trabajo sostenido derivado de la ciencia, educación, conocimiento complementado con políticas de control de la corrupción, fenómeno que según el Banco Mundial compromete el $5 \%$ del PBI mundial ${ }^{1}$.

\section{Gráfico No 01}

PBI de países industrializados y emergentes

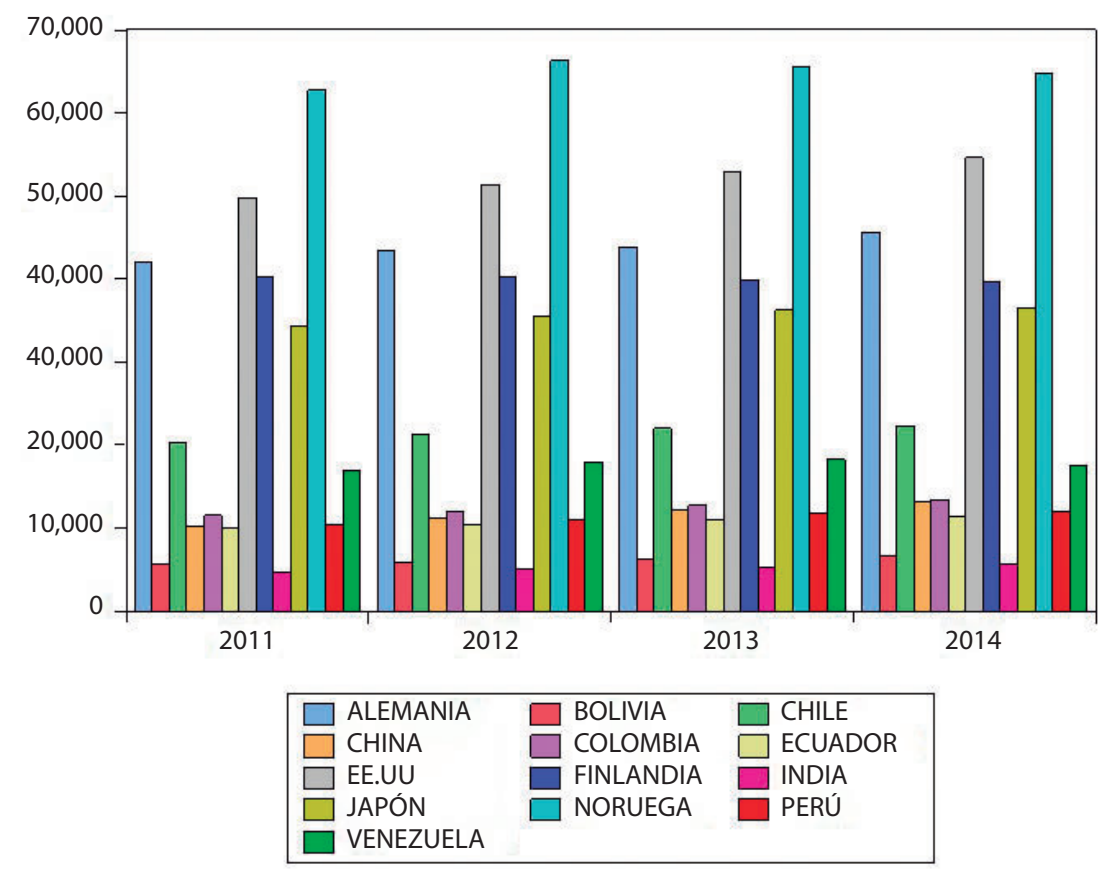

Fuente: Banco Mundial.

\footnotetext{
1 Cifra de Frank Voglha cofundador y asesor de Transparencia Internacional, la organización anticorrupción más grande del mundo; cofundador de Partnership for Transparency Fund; exdirector de Información\&Asuntos Públicos del Banco Mundial y autor del libro Waging War on Corruption - Inside the Movement Speaking Truth to Power.
} 
Existen estudios que demuestran la correlación directa entre la corrupción y la cantidad de recursos naturales que posee un país ${ }^{2}$, factor que se convierten en uno de los principales factores de retardo en el proceso de crecimiento económico. Bajo esta misma línea de análisis cabe precisar que, tomando las cifras de transparencia internacional sobre el ranking de la corrupción global de 2014, en los países en donde se presenta en menor grado la corrupción como Noruega, Dinamarca, Finlandia, Suecia, Nueva Zelanda, Singapur, son los que han logrado mejores resultados macroeconómicos, todo lo contrario sucede con países como Bolivia, Perú, Venezuela, Ecuador, México en donde la corrupción ha sido uno de los impedimentos para lograr su desarrollo. Y qué no decir de la África Subsahariana, catalogada como la región económica con los peores indicadores económicos y sociales pero poseedora de importantes recursos naturales.

Si un país en un largo plazo no quiere estar enclavado a la explotación de recursos naturales, su política pública debe de inclinarse al desarrollo de un sistema educativo inclusivo, el cual debe ser de alta calidad y totalmente democratizada. Tal como argumentan los analistas, los pilares básicos para dar sostenibilidad al proceso de desarrollo es el capital intelectual, o en todo caso en la inversión que los gobiernos puedan realizar en la persona como principal activo para lograr los mejores resultados económicos y sociales. El gráfico $\mathrm{N}^{\circ} 02$ da consistencia a lo afirmado, en donde se aprecia que los países que tienen un pobre desempeño económico son los que menos invierten en investigación y desarrollo, creándose una plataforma debilitada para impulsar el proceso de crecimiento y desarrollo económico basado en franjas industriales en donde predomine el conocimiento. El caso de Perú y Bolivia podría ser las cifras representativas de los países que solo ven a los recursos extractivas como alternativa de crecimiento. Todo lo contrario sucede con Japón, Finlandia, Alemania, Israel, calificados como elites económicas, logro alcanzado a base de invertir en el capital intelectual.

\section{Gráfico No 02}

Inversión en I\&D por países: 2012 (\%)

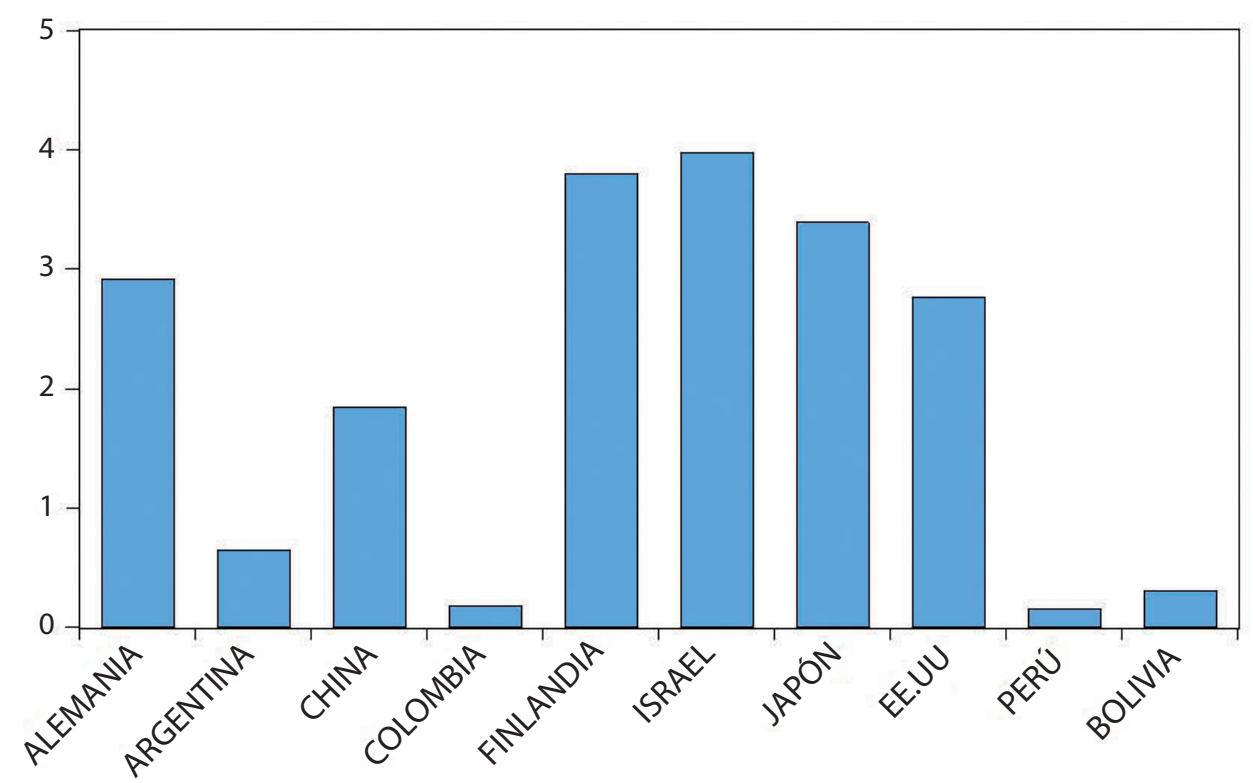

Fuente: Banco Mundial.

2 Tarimo Aquile(2014), editorial verbo divino: El continente africano posee abundantes recursos naturales, y, sin embargo, la mayoría de sus habitantes carecen de un nivel de vida digno. La causa principal de esta injusticia es la corrupción en las instituciones públicas que conceden contratos de explotación minera y asignación de terrenos a inversores extranjeros. Los funcionarios del Estado malversan fácilmente los ingresos por la excesiva centralización del Gobierno, la falta de transparencia burocrática, la impunidad por violar las leyes anticorrupción, y la población que deambula entre la apatía y la desesperanza. La Iglesia católica está llamada a ejercer de líder mediante el ministerio de la justicia social y la formación espiritual y moral de ciudadanos responsables. 


\section{La minería en el Perú y los resultados económicos}

Minería y efectos sociales

¿Cuánto influye las exportaciones en el crecimiento económico del Perú?. En un informe presentado por la Sociedad Nacional de Minería, Petróleo y Energía (SNMPE) en el 2012, se lee lo siguiente: ... los impuestos provenientes de esta actividad tuvieron capacidad para cubrir los gastos del Ministerio de Salud, de Justicia y Derechos Humanos, Mujer y Poblaciones Vulnerables y Trabajo y Promoción Social que en conjunto suma los S/ 9,87 miles de millones..." Como se aprecia en estas cifras, el desarrollo de esta actividad extractiva por los impuestos que paga, es capaz de solventar importantes gastos sociales, bien se podría tomar este hecho como una importante opción de financiamiento para el gobierno, pero también podría ser una vulnerabilidad para los ejecutores de las políticas sociales, ya que la sostenibilidad financiera de las entidades gubernamentales van a estar supeditados a los vaivenes o inestabilidades de los precios de los commodities. En términos más simples, los objetivos y metas sociales estarán ancladas al desarrollo de las empresas mineras y estos a la vez, al mercado internacional, hecho que abre importante espacio de vulnerabilidad en las cuentas presupuestales del gobierno
El colchón financiero que brinda la minería con sus impuestos no solo se da en los sectores sociales ya señalados, sino también, generan importante palanqueo financiero a las inversiones públicas que se ejecutan a nivel local y regional, hecho que se constata con los aportes del Canon y Regalías mineras cuyo objetivo principal es generar espacios de bienestar a la comunidad localizada en la zona de influencia de los proyectos. Claro está que, la eficacia del gasto es discutible en las áreas de influencia, tema que ya no le compete a la sociedad minera como aportante de capital. La evolución del canon minero ha sido importante, si bien, ha generado importantes beneficios comunitarios, pero a la vez, se ha constituido en una fuente de conflicto en los diversos espacios sociales y que se ha esparcido a nivel nacional potenciado por grupos violentistas y radicales.

Aquí, encontramos otro punto de vulnerabilidad, las inversiones públicas financiadas por tributos y no tributos mineros que supeditan el desarrollo de las inversiones gubernamentales, que como se observa en el gráfico $\mathrm{N}^{\circ} 03$, estas han crecido en forma exponencial en los últimos años, gracias a los buenos precios de los minerales en los mercados internacionales. Este grado de dependencia abre otro espacio de vulnerabilidad de los gastos de inversión ante las volatilidades de los precios internacionales.

\section{Gráfico No 03}

Inversiones públicas y tributos mineros como $\%$ del PBI

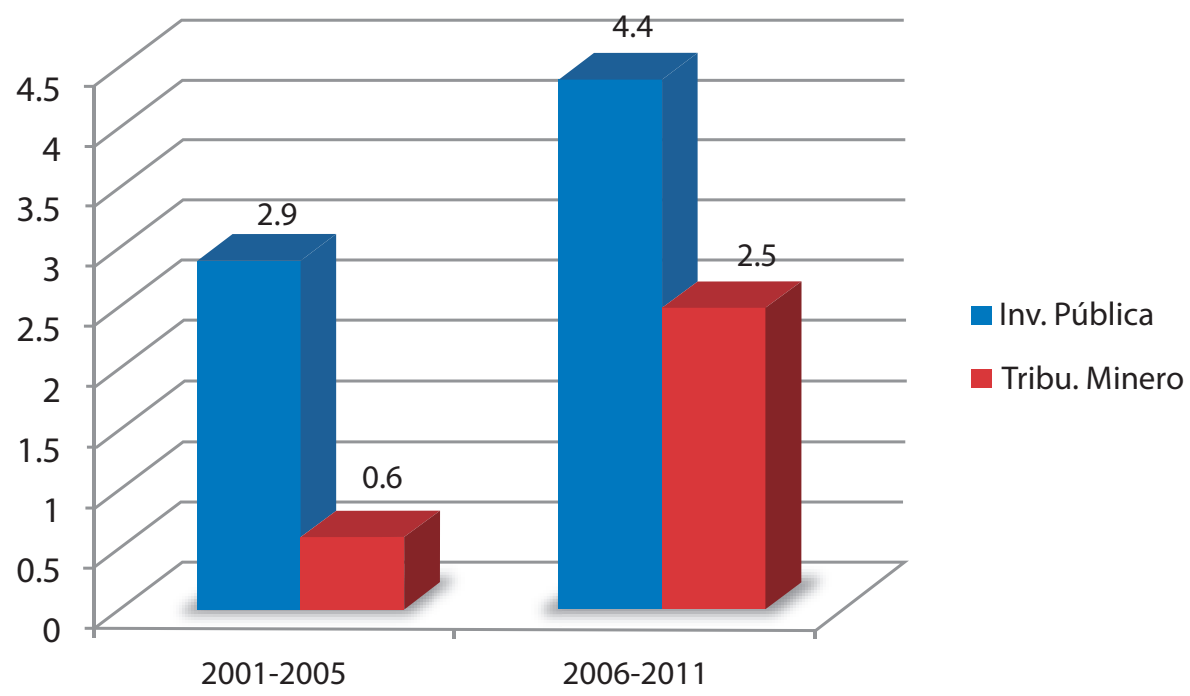

Fuente: MEF. 
Asimismo, en el gráfico $\mathrm{N}^{\circ} 03$ se puede apreciar que en el quinquenio 2001-2005 la brecha entre la inversión pública y los tributos mineros fue del $2.3 \%$, resultado que se acorta en el quinquenio 2006-2011 a un $1.9 \%$, lo cual denota el importante aporte de este sector para el financiamiento de los proyectos de inversión pública. La sostenibilidad de estas inversiones, lo cual implica el aseguramiento de su financiamiento, al igual que otros gastos sociales estará correlacionada al comportamiento del mercado internacional. Que si, la economía mundial, debido al empuje de China y EE.UU logra avances importantes, la robustez tributaria estará asegurada, de suceder todo lo contrario en los mercados globales, el Gobierno Central sentirá el impacto negativo de esta turbulencia al profundizarse los forados fiscales.

Un indicador que podría indicar la fortaleza o debilidad tributaria y que tiene que ver con la actividad minera es el Canon. $\mathrm{Al}$ respecto, la Sociedad Nacional de Minería, Energía y Petróleo (2015) Expresa lo siguiente: “... no olvidemos que el Canon es un fiel reflejo de la rentabilidad del sector (....) Por tal motivo, en momentos de auge se captó mayores recursos vía Impuesto a la Renta, pero debido a la crisis financiera internacional de 2008, las utilidades de las empresas mineras se redujeron y por ende, el Impuesto a la Renta y el Canon Minero. No obstante ello, desde el 2010 se viene recuperando dichas transferencias, aunque con un retroceso en los últimos dos años debido a la caída de las cotizaciones internacionales. En los últimos 19 años se han transferido a los Gobiernos Locales, Regionales, Universidades e Institutos Nacionales más de S/.36,200 millones ...”.

Lo afirmado por esta entidad solo refleja le elevada correlación de los precios internacionales de los minerales con los ingresos tributarios y el subyacente derivado de esta actividad, conocido como el canon minero. Por ello, se argumentaba que las obras de desarrollo regional, aparte de constituirse en una fuente de distorsiones sociales, está supeditada a los eventos internacionales.

\section{Gráfico No 04}

Canon Minero: 1996-2014 (miles soles)

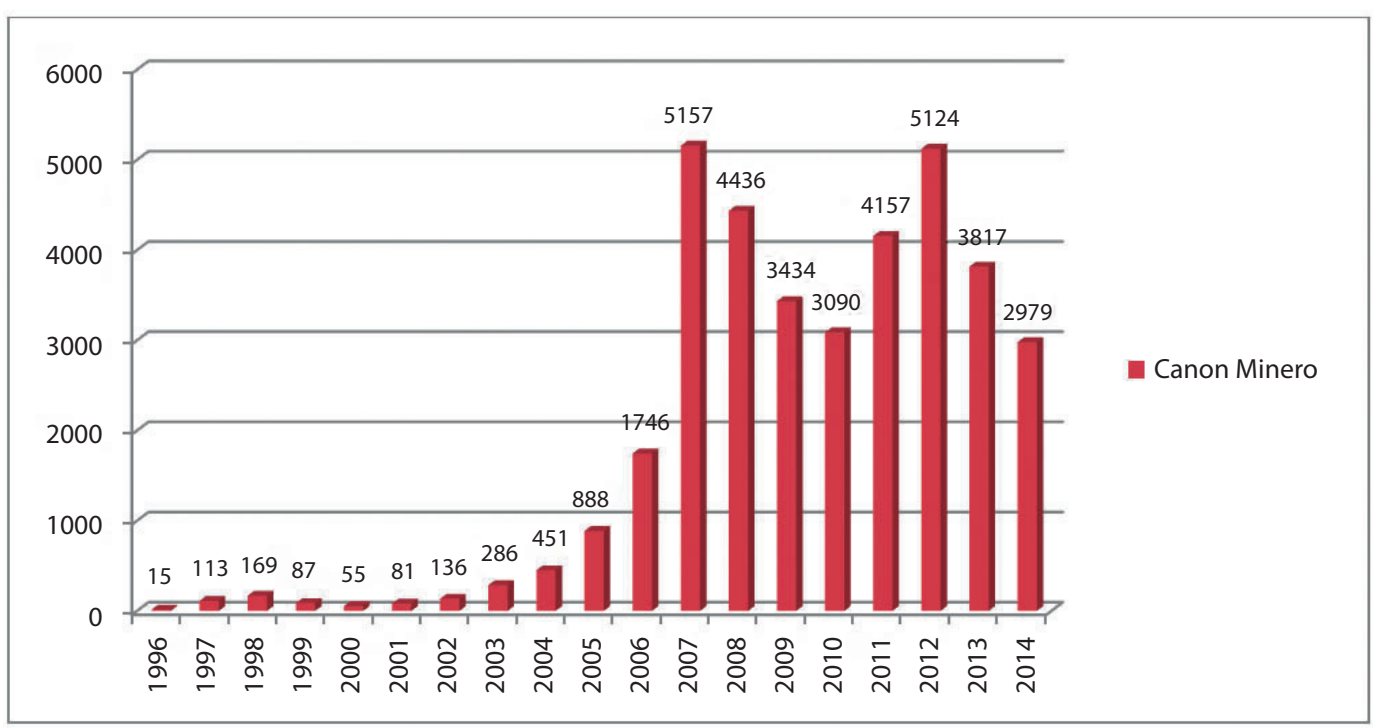

Fuente: Sociedad Nacional de Minería, Energía y Petróleo.

Como se argumentó, en aquellas sociedades en donde su proceso de crecimiento está supeditada a la extracción de recursos naturales como son las mineras, los grupos de poder generan espacios de convulsiones sociales, que se ven traducida inclusive en situaciones de extrema gravedad para los gobiernos democráticos. Bajo el pretexto de las reivindicaciones económicas, grupos organizados asumen posturas de enfrentamientos en contra las organizaciones del Estado. 
El gráfico $\mathrm{N}^{\circ} 05$ expresa claramente lo señalado, en aquellos lugares en donde se encuentran los yacimientos de concentrados, se presentan en mayor grado los conflictos sociales, tales como: Ancash, Apurímac, Cajamarca, Puno, espacios geográficos, ricos en recursos naturales, pero también, castigados por las acciones de convulsiones sociales. El caso extrema es Ancash, que a pesar de captar importante recursos financieros como producto del Canon, su gobierno regional y local, hasta el Central, no han sido capaz de eliminar los síntomas de la extrema pobreza y los estructurales actos de corrupción.

\section{Gráfico No 05}

Conflictos sociales por departamento: 2013

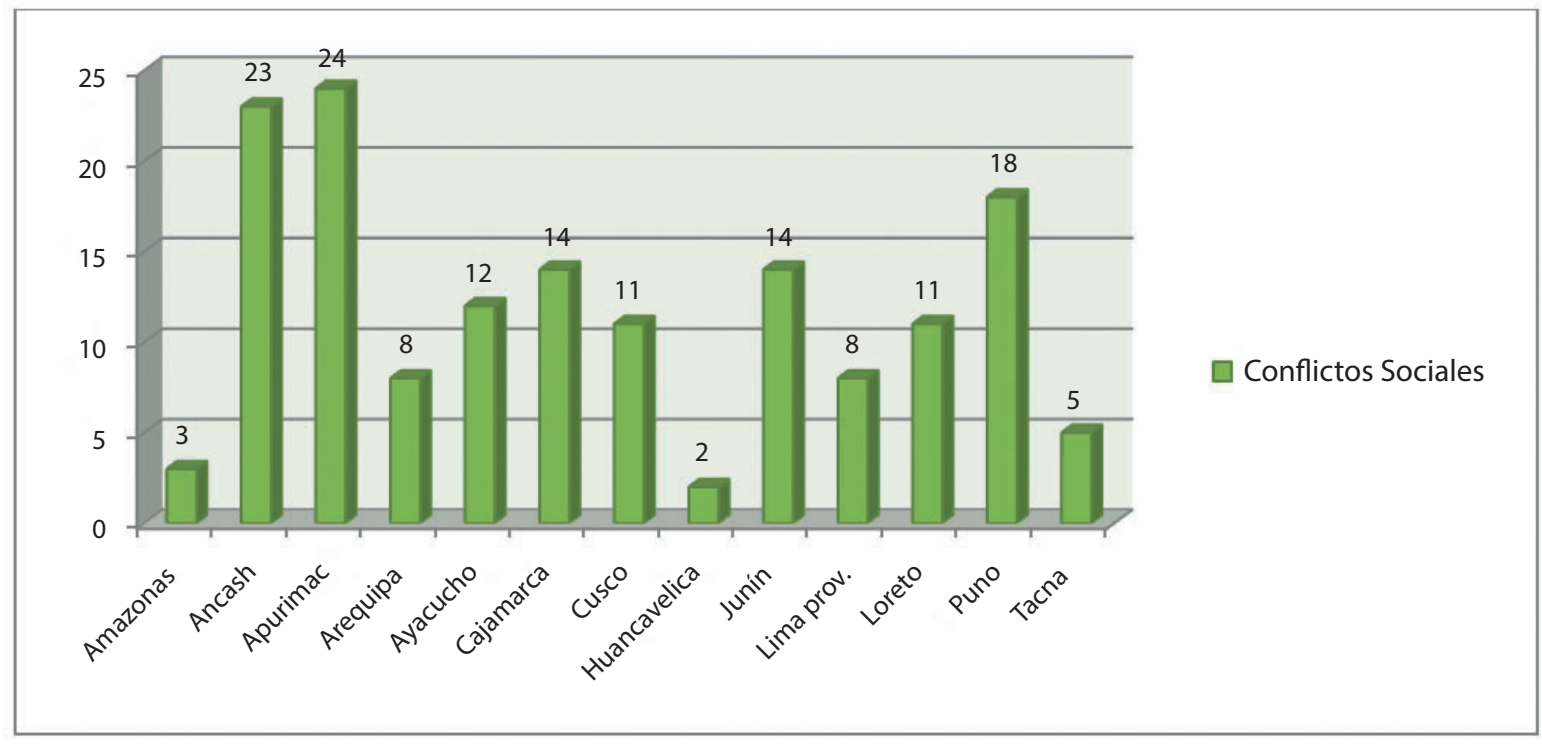

Fuente: Observatorio de Conflictos Sociales.

\section{CONCLUSIONES}

1. Los países que que han potenciado su crecimiento en base a la investigación, innovación, educación, tecnología, entre otros factores, en comparación con los países que han sustentado su crecimiento en los recursos naturales, han logrado en el tiempo mejores resultados económicos y sociales, con indicadores macroeconómicos que se tradujeron en bienestar social.

2. Los ingresos gubernamentales y por ende, los gastos y proyectos sociales, están correlacionados a la tributación de las empresas mineras que son consideradas como principales contribuyentes,lo cual explica el anclaje de la mejora de los indicadores sociales a los vaivenes de los precios internacioanles de los minerales.

3. La actividad extractiva como la minería, en sus respectivas zonas de infuencia, genera graves conflictos sociales, situaciones de crisis que es agravada por los grupos de poder y que vulneran la estabilidad económica y social.

\section{REFERENCIAS BIBLIOGRÁFICAS}

1. SACHS, J. \& WARNER, A. (1997). Natural Resource Abundance and Economic Growth. Harvard University, Cambridge MA.AFIA

2. STIGLITZ, J., SACHS, J. \& HUMPRHEYS, M. (2007) Escapando de la maldición de los recursos naturales.

3. WOOLDRIDGE, J. M. (2007). Introducción a la econometría. Un enfoque moderno (2da. ed.). Madrid: Editorial Thompson.

4. Banco Central de Reserva del Perú (2014): Memorias. Lima Perú

5. Banco Mundial. http://datos.bancomundial.org/ indicador/NY.GDP.PCAP.PP.CD 